\title{
Osteomielite mandibular após extração dentária traumática: Relato de caso
}

\author{
Mandibular osteomyelitis after traumatic tooth extraction: Case report \\ Osteomielitis mandibular tras extracción dentaria traumática: Reporte de un caso
}

Isabella Schefler Henrique Cordeiro

ORCID: https://orcid.org/0000-0003-1858-5409

Universidade Federal de Mato Grosso do Sul, Brasil

E-mail: isabella.henrique@ufms.br

Jose Carlos Garcia de Mendonça

ORCID: https://orcid.org/0000-0003-3217-872X

Universidade Federal de Mato Grosso do Sul, Brasil

E-mail: jose.mendonca@ufms.br

Gustavo Silva Pelissaro

ORCID: https://orcid.org/0000-0003-3475-6001

Universidade Federal de Mato Grosso do Sul, Brasil

E-mail: gustavopelissaro@hotmail.com

Daniella Moraes Antunes

ORCID: https://orcid.org/0000-0002-7557-5461

Universidade Federal de Mato Grosso do Sul, Brasil

E-mail: dani07antunes@gmail.com

Julio Cesar Leite da Silva

ORCID: https://orcid.org/0000-0002-9835-7432

Universidade Federal de Mato Grosso do Sul, Brasil

E-mail: julio.silva@ufms.br

Maisa de Oliveira Aguillera

ORCID: https://orcid.org/0000-0003-0055-6984

Universidade Federal de Mato Grosso do Sul, Brasil

E-mail: maisa.aguillera@live.com

Francielly Thomas Figueiredo

ORCID: https://orcid.org/0000-0003-0196-1172

Universidade Federal de Mato Grosso do Sul, Brasil

E-mail: Francielly.thomas@ufms.br

Ellen Cristina Gaetti Jardim

ORCID: https://orcid.org/0000-0003-2471-465X

Universidade Federal de Mato Grosso do Sul, Brasil

E-mail: ellen.jardim@ufms.br

\begin{abstract}
Resumo
O objetivo do trabalho é relatar um caso de osteomielite crônica em corpo, ramo e cabeça de mandíbula unilateral após exodontia traumática. Paciente do sexo masculino, 45 anos, encaminhado ao Serviço de Cirurgia e Traumatologia Buco-Maxilo-Facial do Hospital Universitário Maria Aparecida Pedrossian, Campo Grande - MS para tratamento de infecção odontogênica acometendo região pré-auricular com extensão bucal e submandibular direita, endurecido à palpação e com fístula extra-oral com drenagem ativa de secreção purulenta. Ao exame intra-oral, alvéolos com sinal de exodontia recente, dentes 47 e 48, e limitação de abertura bucal. Ao exame tomográfico, degeneração óssea de cabeça e ramo mandibular direito compatível com um quadro de osteomielite. Foi realizada drenagem intra e extra-oral com desbridamento da região alveolar dos dentes 47 e 48 e instalação de dreno transfixante sob anestesia local. Aos exames bioquímicos, apresentou quadro de anemia ferropriva, com valores reduzidos de hemoglobina, sugestivo de baixa resposta imunológica levando à disseminação rápida da infecção. Paciente permaneceu 59 dias internado para tratamento medicamentoso via endovenosa (vancomicina, ciprofloxacina e meropenem) para remissão do quadro infeccioso associado a procedimentos de cultura, antibiograma e curetagem da região de corpo mandibular. Após o tratamento mencionado o paciente apresentou resposta satisfatória com remissão da sintomatologia e seguiu em acompanhamento ambulatorial sem queixas e/ou complicações.
\end{abstract}

Palavras-chave: Osteomielite; Diagnóstico; Mortalidade; Tratamento farmacológico.

\section{Abstract}

The objective of this study is to report a case of chronic osteomyelitis in the unilateral body, branch and head of the jaw after traumatic extraction. Male patient, 45 years old, referred to the Oral-Maxillofacial Surgery and Traumatology Service of Maria Aparecida Pedrossian's University Hospital in Campo Grande - MS for the treatment of odontogenic infection affecting the pre-auricular region with right oral and submandibular extension, hardened on 
palpation and with extraoral fistula with active drainage of purulent secretion. On intraoral examination, alveoli with signs of recent extraction, teeth 47 and 48, and limitation of mouth opening. On tomography, bone degeneration of the head and right mandibular branch compatible with osteomyelitis. Intraoral and extraoral drainage was performed with debridement of the alveolar region of teeth 47 and 48 and installation of a transfixing drain under local anesthesia. On biochemical examinations, he presented iron deficiency anemia, with reduced hemoglobin values, suggestive of low immune response leading to rapid spread of infection. Patient remained hospitalized for intravenous drug treatment for 59 days (vancomycin, ciprofloxacin and meropenem) for remission of the infectious condition associated with culture procedures, antibiogram and curettage of the mandibular body region. After the mentioned treatment, the patient presented a satisfactory response with remission of symptoms and continued in outpatient follow-up without complaints and/or complications.

Keywords: Osteomyelitis; Diagnosis; Mortality; Drug therapy.

\section{Resumen}

El objetivo de este estudio es reportar un caso de osteomielitis crónica en cuerpo unilateral, rama Y cabeza de la mandíbula tras extracción traumática. Paciente varón de 45 años, remitido al Servicio de Cirugía y Traumatología Oral-Maxilofacial del Hospital Universitario Maria Aparecida Pedrossian de Campo Grande - MS, para el tratamiento de una infección odontogénica que afecta a la región preauricular con extensión oral Y submandibular derecha, endurecida a la palpación. Con fístula extraoral con drenaje activo de secreción purulenta. Al examen intraoral, alvéolos con signos de extracción reciente, dientes 47 Y 48, limitación de la apertura bucal. En la tomografía, degeneración ósea de la cabeza Y rama mandibular derecha compatible con osteomielitis. Se realizó drenaje intraoral Y extraoral con desbridamiento de la región alveolar de los dientes 47 Y 48 e instalación de un drenaje transfijante bajo anestesia local. En los exámenes bioquímicos presentaba anemia ferropénica, con valores reducidos de hemoglobina, sugestivos de baja respuesta inmune que conducía a una rápida propagación de la infección. El paciente permaneció hospitalizado para tratamiento farmacológico intravenoso durante 59 días (vancomicina, ciprofloxacina Y meropenem) por remisión de la patología infecciosa asociada a procedimientos de cultivo, antibiograma Y legrado de la región del cuerpo mandibular. Tras el tratamiento mencionado, el paciente presentó una respuesta satisfactoria con remisión de los síntomas y continuó en seguimiento ambulatorio sin quejas Y/o complicaciones.

Palabras clave: Osteomielitis; Diagnóstico; Mortalidad; Tratamiento farmacológico.

\section{Introdução}

Infecções odontogênicas são relativamente comuns em região de cabeça e pescoço, tratadas na maioria dos casos de forma simples com prognóstico favorável (Lima et al., 2014). Porém, na presença de um desequilíbrio entre sistema imune do hospedeiro e a virulência microbiana, predispõe-se a uma infecção severa, podendo evoluir para comprometimento sistêmico além do sítio de origem, a partir do envolvimento de espaços medulares e sistema circulatório haversiano, com disseminação além do periósteo nos espaços fasciais (Miloro et al., 2016; Masocatto et al., 2017).

A osteomielite é conceituada como uma inflamação no osso medular podendo se estender ao periósteo da área comprometida elevando-o do córtex, o que sucede em falta de fornecimento sanguíneo para o osso local, favorecendo o desenvolvimento de microrganismos (Masocatto et al., 2017). Assim sendo, a mandíbula é mais acometida, por apresentar menor aporte sanguíneo em relação à maxila (Topazian et al., 1997). A patogênese pode estar ligada à disseminação local de microrganismos externos a microbiota corporal, presentes em processos infecciosos adjacentes, notadamente as infecções odontogênicas e, em especial, infecções endodônticas (Masocatto et al., 2017; Brook \& Frazier, 1993).

Desta maneira, a osteomielite pode se apresentar como processo agudo e crônico. Na osteomielite aguda, ocorre um processo inflamatório não circunscrito, de rápido curso evolutivo, associado à febre, leucocitose, linfadenopatia, dor e tumefação e sem qualquer significância radiográfica (Kim et al., 2001; Reinert et al., 1999; Wakasa et al., 2002). Já a osteomielite crônica pode ser primária, surgindo como infecção de menores proporções clínicas além de atenuação dos sinais flogísticos principais como dor branda e presença de sequestros ósseos (Lima et al., 2014) ou secundária, ocorrendo a partir de uma osteomielite aguda que não foi tratada ou recebeu inadequada terapêtica (Koorbusch et al., 1992). Por se tratar de um curso de evolução mais lento, passa a ter características imaginológicas, como o surgimento da radiolucidez com padrão uniforme ou salpicado, como roído de traça (Masocatto et al., 2017). 
Os principais fatores predisponentes são virulência do microrganismo, resistência do hospedeiro, doenças sistêmicas, desnutrição, assim como condições que alteram a vascularização (Lima et al., 2014). Em especial, a deficiência de ferro também pode gerar anormalidades epiteliais e comprometer a imunidade, com redução na eficiência bactericida de leucócitos polimorfonucleares e resposta inadequada de anticorpos (Licciardello et al., 2007).

O tratamento mais eficaz para osteomielite consiste na remoção da causa da infecção associada à antibioticoterapia. Normalmente a terapêtica compreende na remoção dos sequestros ósseos, desbridamento da lesão, decorticação óssea associada ao emprego sistêmico de antimicrobianos, geralmente de amplo espectro de ação (Masocatto et al., 2017; Brook \& Frazier, 1993; Lew \& Waldvogel, 1997).

Logo, esse trabalho tem como objetivo relatar um caso de osteomielite de mandíbula após extração dentária traumática em um homem de 45 anos, portador de sífilis e anemia ferropriva, em que o mesmo extraiu o próprio dente, necessitando de uma abordagem multiprofissional.

\section{Metodologia e Relato de Caso}

O presente caso trata-se de um relato com fins descritivos, exploratórios e com abordagem qualitativa, a fim de mostrar sua relevância clínica e facilitar pesquisas e novos relatos com a mesma temática, sempre baseada em evidência, usando como base referencial Pereira (2018) e Souza et al. (2021). O trabalho recebeu anuência dos genitores do paciente após assinatura do Termo de Consentimento Livre e Informado para participação. Foram respeitados todos os princípios éticos na condução do tratamento do paciente.

Paciente do gênero masculino, 45 anos, encaminhado ao Serviço de Cirurgia e Traumatologia Buco-Maxilo-Facial do Hospital Universitário Maria Aparecida Pedrossian de Campo Grande-MS, relatando que extraiu dentes em mandíbula em sua casa com o uso de alicate comum há 9 dias da procura pelo serviço. Apresentou desde então, aumento volumétrico, queixas de dor na região mandibular e ouvido do lado direito, além de limitação de abertura bucal. Durante a anamnese, negou comorbidades sistêmicas e uso de medicamentos de rotina bem como, etilismo, tabagismo ou uso de outras drogas.

Ao exame físico, foi observado aumento volumétrico endurecido em região pré-auricular estendendo-se a região bucal do lado direito com leve acometimento da região submandibular e presença de fístula extraoral com drenagem ativa de exsudato purulento. Ao exame intraoral região dos alvéolos dos dentes 47 e 48 também com drenagem de exsudato durante manipulação e destruição coronária do elemento 37. Notou-se também, alvéolos dos elementos 47 e 48 sem alterações dignas de nota e elemento 37 com presença de reabsorção perirradicular ao exame de imagem. Sendo assim o paciente recebeu o diagnóstico de infecção de origem odontogênica condizente com o quadro de osteomielite crônica (Figura 1). 
Figura 1: Aspectos clínicos e tomográficos. Paciente com drenagem espontânea de coleção purulenta em região submandibular à direita com instalação de dreno de Penrose intrabucalmente. Presença de pequenos fragmentos ósseos em região posterior de mandíbula a direta.
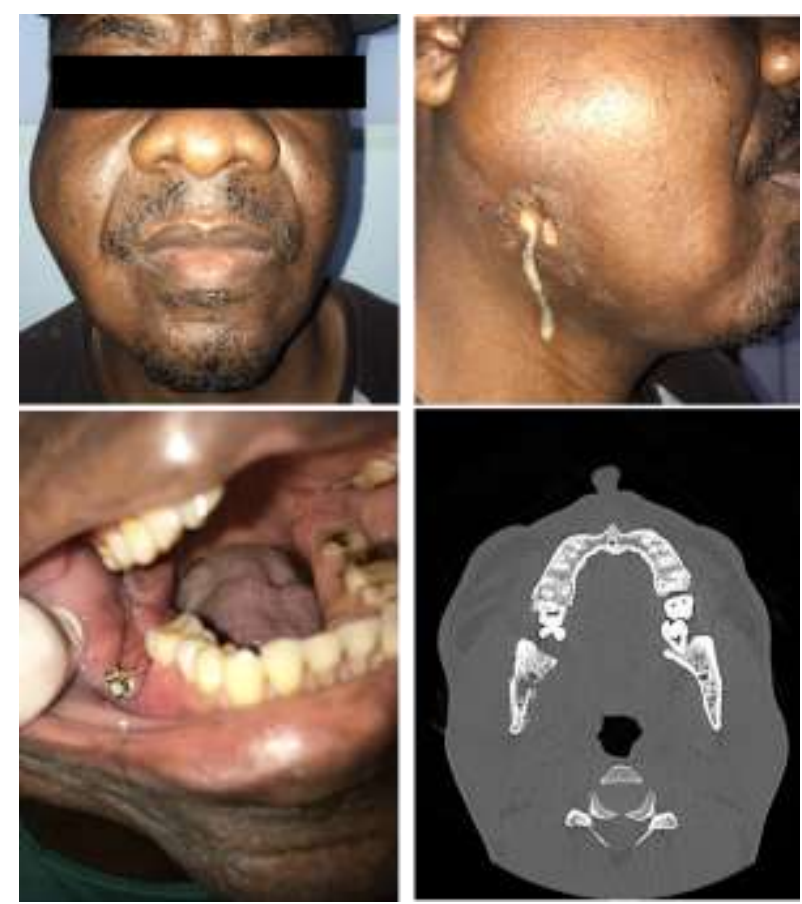

Fonte: Autores.

Com exame PCR (Proteína C-Reativa) no valor de 131,37, realizou-se internação hospitalar, antibioticoterapia com administração endovenosa de ampicilina $1 \mathrm{~g}$ de 8 em 8 horas e metronidazol $500 \mathrm{mg}$ de 12 em 12 horas, abertura de alvéolo em região do dente 48, curetagem, coleta de material para análise de cultura e antibiograma, regularização do rebordo alveolar, remoção de espículas ósseas presentes ao redor deste alvéolo, irrigação com iodopovidona (PVPI) e soro fisiológico 0,9\% e sutura com nylon 4-0. O procedimento foi realizado sob anestesia local, sem intercorrências.

Aos exames bioquímicos observou-se redução significativa na concentração de hemoglobina, VCM (Volume Corpuscular médio) e plaquetose, resultantes de deficiência de ferro e compatível com quadro de anemia ferropriva. O paciente, ainda hospitalizado, recebeu avaliação da equipe de hematologia que prescreveu sulfato ferroso, duas cápsulas, duas vezes ao dia.

Com exame de sorologia, VDRL (Estudo Laboratorial de Doenças Venéreas) positivo, paciente foi também avaliado pela equipe de infectologia que diagnosticou o quadro como sífilis latente tardia, tendo a conduta administração de Penicilina Benzatina 1.200.000 UI em cada glúteo, uma vez por semana, durante três semanas.

Após 16 dias de internação com tratamento antimicrobiano empírico, acompanhamento médico e solicitação de antibiograma, o paciente apresentou evolução em todas as condições, tanto da infecção bucal quanto da sua condição sistêmica, melhora de queixas álgicas, ausência de exsudato purulento extraoral e em região dos dentes extraídos, suturas em posição, seguindo então de alta hospitalar.

Em retorno pós operatório de 5 dias, paciente apresentou drenagem ativa de exsudato purulento intra e extraoral, sendo realizada nova abordagem cirúrgica para instalação de dreno de penrose transfixante submandibular do lado direito e solicitação de exames complementares de cultura e antibiograma que evidenciaram resistência bacteriana. Em resultado de primeira cultura detectou-se Streptococcus oralis, com sensibilidade intermediária às ampicilinas, sensibilidade à gentamicina, 
meropenem, teicoplanina e vancomicina e resistente às cefalosporinas. A partir disso, o antibiótico de escolha foi a vancomicina 900mg com via de administração endovenosa, necessitando então de nova admissão hospitalar.

Após nova internação, em exame tomográfico e cintilografia óssea, observou-se degeneração óssea em côndilo direito e regiões alveolar e lingual, ambas acometidas pela infecção odontogênica. Foram solicitados novos exames bioquímicos e parecer da reumatologia para investigação de possíveis causas, que descartaram doenças degenerativas sugerindo quadro compatível de osteomielite ocasionado por infecção de origem odontogênica (Figura 2).

Figura 2: Reconstruções tomográficas evidenciando degeneração óssea em côndilo direito e regiões de alvéolo e corticais ósseas mandibulares ipsilaterais.
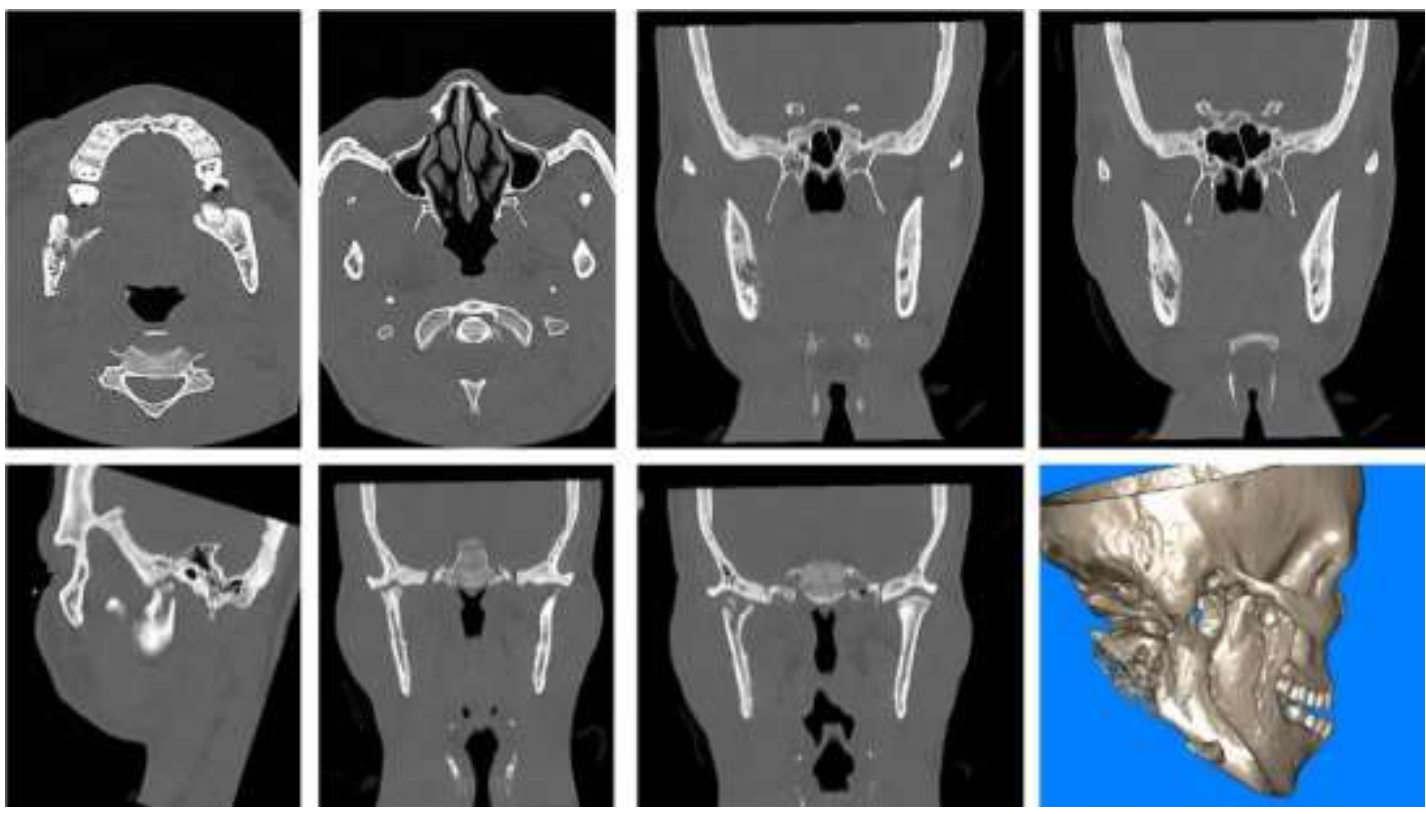

Fonte: Autores.

Clinicamente, paciente ainda apresentava drenagem ativa de exsudato purulento intra e extraoral, sendo realizadas lavagens diárias do dreno com soro fisiológico, PVPI e troca de curativos. Realizou-se inserção de novo dreno e coleta de material. O resultado do exame de cultura, detectou a presença de Pseudomonas aeroginosa; assim a conduta tomada pela equipe médica em conjunto com a equipe Buco-Maxilo-Facial foi de administração do antibiótico ciprofloxacino 400mg de 8 em 8 horas e posteriormente substituição por meropenem $1 \mathrm{~g}$ de 8 em 8 horas endovenoso, por apresentar reações inerentes ao antibiótico supracitado como êmese e dor abdominal. Após exames realizados pela clínica médica, paciente foi diagnosticado com esofagite, para a qual foi administrado omeprazol 40mg de $12 \mathrm{em} 12$ horas.

Após melhora clínica e laboratorial, o paciente foi submetido à exodontia do elemento 37. Após melhora nos exames bioquímicos e no quadro clínico geral, o paciente não apresentou mais queixas, sejam elas álgicas e/ou funcionais, com melhora de abertura bucal, movimentos mandibulares preservados e fechamento de fístula extraoral. Depois de 59 dias, com 42 dias do novo ciclo antimicrobiano, o paciente recebeu alta hospitalar (Figura 3). 
Figura 3: Vista frontal e lateral de face à direita. Diminuição acentuada do edema em hemiface direita e fechamento de fístula submandibular do mesmo lado.

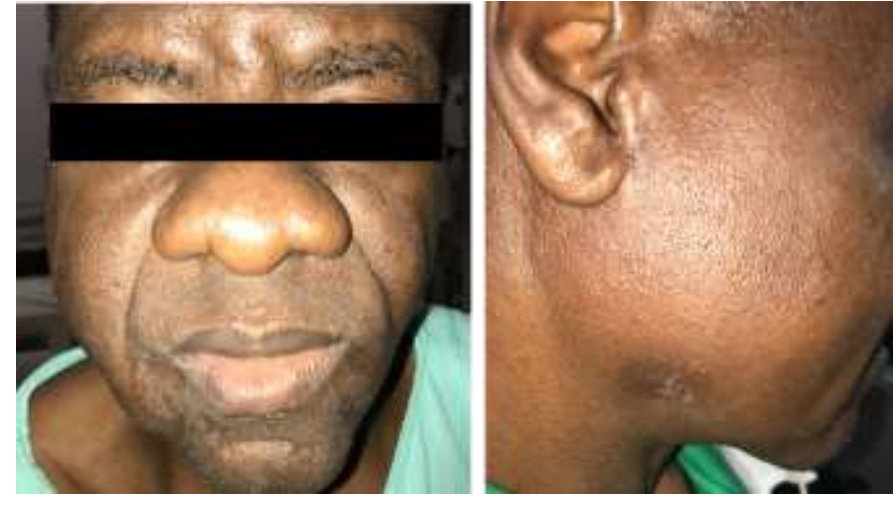

Fonte: Autores.

\section{Discussão}

Dentre os fatores locais de desenvolvimento da osteomielite, destacam-se as infecções odontogênicas que evoluem, sobretudo, a partir do comprometimento sistêmico do paciente. A evolução da infecção se dá quando microrganismos entram na medula óssea gerando uma resposta inflamatória, e assim, reabsorção óssea, perda de osso cortical acompanhado de dor, edema, febre e linfadenopatia, como também, drenagem de coleção purulenta (Mardini \& Gohel, 2018). Tal fato vem ao encontro do caso retratado, uma vez que o próprio paciente extraiu o elemento dentário, tendo como consequência infecção odontogênica que por conta das alterações do estado de saúde, baixa imunidade, anemia ferropriva e sífilis latente tardia, o quadro evoluiu para degeneração óssea em cabeça da mandíbula à direita e ramo mandibular ipsilateral.

A osteomielite é uma doença heterogênea em sua fisiopatologia, apresentação clínica e manejo terapêutico. Em virtude da característica multifatorial - microbiota alterada, resistência dos microrganismos a vários antibióticos e presença de fatores predisponentes (Masocatto et al., 2017), essa enfermidade configura como uma das doenças infecciosas mais difíceis de tratar (Sia \& Berbari, 2006). Assim, o caso relatado corrobora com a literatura, visto que o paciente não apresentou resposta à antibioticoterapia empírica inicial, por resistência microbiana, associada ao contexto de desordens sistêmicas graves, a desnutrição e dificuldade de autocuidado demonstrada no momento da internação hospitalar.

Embora a Organização Mundial da Saúde (2020) cite a anemia como a redução de hemoglobina na circulação sanguínea, sobretudo de pacientes de tenra idade e mulheres grávidas, seja hereditária ou por deficiência nutricional, coloca em evidência um problema global: a desnutrição do paciente aqui abordado, do gênero masculino, de 45 anos de idade, o que vai contra ao evidenciado pela OMS. Tal abordagem se revela importante uma vez que, como já mencionado, o tratamento da osteomielite crônica não é simples e o diagnóstico da mesma necessita ser rápido e preciso. Ele envolve um conjunto de sinais e sintomas clínicos além de exames laboratoriais. Geralmente o marcador inflamatório PCR costuma estar elevado, apesar de ser um teste inespecífico, é utilizado para monitorar a evolução do tratamento da infecção (Lima et al., 2014). Além disso, testes histopatológicos e exames de imagem, destacando melhores resultados as tomografias computadorizadas, exames de medicina nuclear os quais se enquadram as cintilografias ósseas e ressonância magnética também são utilizados (Lima et al., 2014). Nesse caso, os exames complementares coadjuvantes à análise e evolução clínica foram essenciais para o correto diagnóstico e conduta cirúrgico-terapêutica condizente com o tempo longo de hospitalização.

Os princípios do tratamento consistem em estabelecer uma terapia antibiótica empírica com base nos organismos infecciosos habituais para a situação clínica em questão, seguida de terapia definitiva com base nos resultados da cultura e testes de sensibilidade (Lew \& Waldvogel, 1997; Lipsky \& Berendt, 2010). O ideal é que se prescrevam doses que garantam 
níveis séricos adequados dos antimicrobianos, considerando inicialmente a terapia intravenosa, favorecendo os agentes com efetividade clínica comprovada (Masocatto et al., 2017; Brook \& Frazier, 1993). Em consonância, deve-se associar aos antimicrobianos a terapia cirúrgica, com drenagem de abscessos em tecidos moles e/ou ósseos, com desbridamento tecidual, ressecção óssea das regiões necrosadas e revascularização (Lew \& Waldvogel, 1997; Sia \& Berbari, 2006), sempre aliados a medidas de suporte como, otimização da condição nutricional e metabólica do paciente, tratamento de distúrbios subjacentes, fornecimento de suporte psicológico, social e de reabilitação de acordo com a necessidade (Lipsky \& Berendt, 2010). A dificuldade descrita acima mostra- se evidente quando é mencionado a dificuldade em debelar a infecção em um organismo com menor aporte sanguíneo local, correlacionando os exames complementares de sangue, uma linha de defesa imunológica deficitária e um quadro de anemia sério com instabilidade hemodinâmica (Silva et al., 2020).

Ainda, no tocante aos antibacterianos, a vancomicina é indicada para o tratamento de infecções graves, corroborando com a clínica acima, causadas por microrganismos gram-positivos suscetíveis que não respondem a outras drogas, incluindo penicilinas, demonstrando efetividade no tratamento de infecções ósseas (Désévaux et al., 2002). Da classe dos carbapenêmicos, o meropenem tem amplo espectro de ação, abrange gram-positivos e negativos, com maior ênfase para estes, também optado para uso em ambientes hospitalares, em situações de resistência bacteriana, como em Pseudomonas aeroginosa, comumente resistente aos antibióticos de uso rotineiro, sendo o tratamento idealmente realizado com a combinação de antibióticos como ciprofloxacina (Maffulli et al., 2016). A grande dificuldade de tratamento no caso descrito se dá pela infecção de uma cepa multirresistente associada à morbidades, como anemia e sífilis, que podem ter interferido diretamente na imunossupressão do paciente, consequentemente, acarretando longa permanência de internação e elevados custos para o sistema de saúde.

De modo geral, o tratamento da osteomielite consiste em uma terapia antibiótica empírica eficaz e tratamento cirúrgico também eficaz, o que demonstra que o tratamento inicialmente proposto estava correto. Preconiza-se o tratamento cirúrgico de forma mais agressiva, devido à característica igualmente agressiva da lesão, o que muitas vezes gera morbidades ao paciente no que tange a função mastigatória do mesmo, com perda de grandes quantidades do arcabouço ósseo facial (Baur et al., 2015). Outros defendem o uso de antimicrobianos de amplo espectro associado a técnicas cirúrgicas menos invasivas ou ausentes, mostrando resultados satisfatórios. Porém, diante das desordens sistêmicas do paciente que interferem na sua imunidade prejudicando a eficácia das terapias, é importante lançar mão de recursos como cultura e antibiograma para teste da susceptibilidade dos microrganismos envolvidos como garantia de uma terapia eficiente (González-Navarro et al., 2017). Desta feita, o caso apresentando, apesar do grande envolvimento lítico da porção ascendente da mandíbula e envolvimento condilar, a terapia cirúrgica restrita ao processo alveolar e drenagem associada a terapia antibiótica guiada pela cultura e antibiograma apresentou resultados satisfatórios, evitando morbidades e garantindo a funcionalidade mastigatória do paciente.

Uma história patológica pregressa meticulosa e uma história social bem feita detectam comorbidades clínicas e propiciam parâmetros claros de tratamento (Miloro et al., 2016). Traçar o perfil do paciente, antes de qualquer procedimento cirúrgico, possibilita a estratificação do mesmo de acordo com o risco cirúrgico e a melhor terapêutica (Miloro et al., 2016; González-Navarro et al., 2017). Quando condições clínicas e comorbidades são reconhecidas, torna-se mais fácil uma investigação diagnóstica apropriada e a participação de outros profissionais de saúde deve ser solicitada quando houver indicação (Miloro et al., 2016). O caso demonstra a importância da boa comunicação multiprofissional, algo extremamente dificultado no caso apresentado pelo paciente falar apenas o idioma de origem e pouco entender de outros, o que prejudicou conseguir informações básicas como sintomatologia pregressa, durante e após tratamento e que poderia ter ajudado de forma mais rápida a melhora do quadro clínico geral e bom desfecho do caso, conseguido às custas de um tempo de internação prolongado e um tratamento exaustivo para o paciente. 


\section{Conclusão}

O tratamento da osteomielite é desafiador e requer abordagens abrangentes e multidisciplinares. O presente caso demonstra o resultado de um controle sistêmico deficitário em um organismo imunossuprimido com resposta pobre a uma infecção odontogênica grave. Foi extremamente desafiador; a resposta, embora cansativa ao paciente, ocorreu pela necessidade de longa internação hospitalar, o que se mostrou dentro do esperado. O paciente segue bem, sem queixas, o que configura sucesso até o momento. O caso apresenta suas limitações por ser único e assim sendo, é importante que novos casos semelhantes sejam agrupados e comparados para aumentar a viabilidade estatística dos resultados alcançados, uma vez que a presença de apenas um plano de tratamento como o mencionado, mesmo que com sucesso, é restrito a reduzida confiança estatística.

\section{Agradecimentos}

O presente trabalho foi realizado com apoio da Fundação Universidade Federal de Mato Grosso do Sul -UFMS/MEC - Brasil.

\section{Referências}

Baur, D. A., Altay, M. A., Flores-Hidalgo, A., Ort, Y., \& Quereshy, F. A. (2015). Chronic osteomyelitis of the mandible: diagnosis and management-an institution's experience over 7 years. Journal of Oral and Maxillofacial Surgery. 73(4):655-65. 10.1016/j.joms.2014.10.017.

Brook, I., \& Frazier, E, H. (1993). Anaerobic osteomyelitis and arthritis in a military hospital: a 10-year experience. The American Journal of Medicine. 94(1):21-8.

Désévaux, C., Lenaerts, V., Girard, C., \& Dubreuil, P. (2002). Characterization of crosslinked high amylose starch matrix implants. 2. In vivo release of ciprofloxacin. Journal of Controlled Release. 18;82(1):95-103. 10.1016/s0168-3659(02)00132-3.

González-Navarro, B., Arranz-Obispo, C., Albuquerque, R., Jané-Salas, E., \& López-López, J. (2017). Osteomyelitis of the jaw (with pathological fracture) following extraction of an impacted wisdom tooth. A case report. Journal of Stomatology, Oral Maxillofacial Surgery. 118(5):306-309. 10.1016/j.jormas.2017.05.003.

Kim, S. G., \& Jang, H. S. (2001). Treatment of chronic osteomyelitis in Korea. Oral Surgery, Oral Medicine, Oral Pathology, Oral Radiology, Endodontology. 92(4):394-8.

Koorbusch, G. F., Fotos, P., \& Goll, K. T. (1992). Retrospective assement of osteomyelitis. Oral Surgery, Oral Medicine, Oral Pathology. 74(2):149-54.

Lew, D. P., \& Waldvogel, F. A. (1997.) Osteomyelitis. The New England Journal of Medicine. 336(14):999-1007.

Licciardello, V., Bertuna, G., \& Samperi, P. (2007). Craniofacial morphology in patients with sickle cell disease: a cephalometric analysis. European Journal of Orthodontics. 29(3): 238-42.

Lima, A. L., Oliveira, P. R., Carvalho, V. C., Cimerman, S., \& Savio, E. (2014). Diretrizes Panamericanas para el Tratamiento de las Osteomielitis e Infecciones de Tejidos Blandos Group. Recommendations for the treatment of osteomyelitis. The Brazilian Journal of Infectious Diseases. 18(5):526-34.

Lipsky, B. A., \& Berendt, A. R. 2010. Osteomyelitis. ACP Medicine. 1-20.

Maffulli, N., Papalia, R., Zampogna, B., Torre, G., Albo, E., \& Denaro, V. (2016). The management of osteomyelitis in the adult. Surgeon. 14(6):345-360. 10.1016/j.surge.2015.12.005.

Mardini, S., \& Gohel, A. (2018). Imaging of Odontogenic Infections. Radiologic Clinics of North America. 56(1):31-44. 10.1016/j.rcl.2017.08.003.

Masocatto, D. C., Oliveira, M. M., \& Mendonça, J. C. G. (2017). Osteomielite crônica mandibular: relato de caso. Archives of Health Investigation. 6(2):4852.

Miloro, M., Ghali, G. E., Larsen, P. E. \& Waite, P. D. (2016). Princípios de cirurgia bucomaxilofacial de Peterson. 3. ed. São Paulo: Santos Editora. 1344 p.

Organização Mundial da Saúde. (2020). Anaemia. https://www.who.int/health-topics/anaemia.

Pereira, P. A., Shitsuka, D. M., Parreira, F. J. \& Shitsuka, R. (2018). Metodologia da pesquisa científica UFSM, NTE. https://repositorio.ufsm.br/bitstream/handle/1/15824/Lic_Computacao_Metodologia-Pesquisa-Cientifica.pdf?sequence=1 .

Reinert, S., Widlitzek, H., \& Venderink, D. J. (1999). The value of the magnetic resonance imaging in the diagnosis of mandibular osteomyelitis. British Journal of Oral and Maxilllofacial Surgery. 37(6):459-63. 
Research, Society and Development, v. 11, n. 1, e28411124815, 2022

(CC BY 4.0) | ISSN 2525-3409 | DOI: http://dx.doi.org/10.33448/rsd-v11i1.24815

Sia, I. G., \& Berbari, E. F. (2006). Osteomyelitis. Best Practice \& Research: Clinical Rheumatology. 20(6): 1065-1081.

Silva, I. L., de Alencar, L. B. B., de Sousa, S. C. A., de Araújo, O. S. M., Moura, A. B. R., de Medeiros, L. A. D. M. et al. Research, Society and Development. 9(7): e619974522, 2020. http://dx.doi.org/10.33448/rsd-v9i7.4522.

Souza , Y. D., Seco, A. J. L. G., Silva, J. C. L. Da, Pereira, K. F. S., Jardim, E. C. G. (2021). Kaposi's Sarcoma in an HIV and Covid-19 positive patient. Research, Society and Development. 10(11): e19101119149. 10.33448/rsd-v10i11.19149.

Topazian, R. G., \& Goldberg, M. H. ;(1997). Infecções maxilofacias e orais. Santos.

Wakasa, T., Higuchi, Y., Hisatomi, M., Aiga, H., Honda, Y., \& Kishi, K. (2002). Application of dynamic CT for various diseases in the oral and maxillofacial region. European Journal of Radiology. 44(1):10-5. 